\title{
Evidence for a Smooth Onset of Deformation in the Neutron-Rich Kr Isotopes
}

M. Albers, ${ }^{1,2, *}$ N. Warr, ${ }^{1}$ K. Nomura,${ }^{3}$ A. Blazhev, ${ }^{1}$ J. Jolie, ${ }^{1}$ D. Mücher, ${ }^{4}$ B. Bastin,${ }^{5}$ C. Bauer, ${ }^{6}$ C. Bernards, ${ }^{1}$ L. Bettermann, ${ }^{1}$ V. Bildstein,,${ }^{4,7}$ J. Butterworth, ${ }^{8}$ M. Cappellazzo, ${ }^{1}$ J. Cederkäll, ${ }^{9}$ D. Cline, ${ }^{10}$ I. Darby, ${ }^{5}$ S. Das Gupta,${ }^{11}$ J. M. Daugas, ${ }^{12}$ T. Davinson, ${ }^{13}$ H. De Witte,${ }^{5}$ J. Diriken $,{ }^{5}, 14$ D. Filipescu, ${ }^{15}$ E. Fiori, ${ }^{16}$ C. Fransen, ${ }^{1}$ L. P. Gaffney, ${ }^{17}$ G. Georgiev, ${ }^{16}$ R. Gernhäuser, ${ }^{4}$ M. Hackstein, ${ }^{1}$ S. Heinze, ${ }^{1}$ H. Hess, ${ }^{1}$ M. Huyse,${ }^{5}$ D. Jenkins, ${ }^{8}$ J. Konki, ${ }^{18}$ M. Kowalczyk ${ }^{19}$ T. Kröll, ${ }^{6}$ R. Krücken, ${ }^{4,20}$ J. Litzinger, ${ }^{1}$ R. Lutter, ${ }^{21}$ N. Marginean, ${ }^{15}$ C. Mihai,${ }^{15}$ K. Moschner, ${ }^{1}$ P. Napiorkowski, ${ }^{19}$ B. S. Nara Singh, ${ }^{8}$ K. Nowak, ${ }^{4}$ T. Otsuka,${ }^{3,22,23}$ J. Pakarinen, ${ }^{9}$ M. Pfeiffer, ${ }^{1}$ D. Radeck, ${ }^{1}$ P. Reiter, ${ }^{1}$ S. Rigby, ${ }^{17}$ L. M. Robledo, ${ }^{24}$ R. Rodríguez-Guzmán, ${ }^{25}$ M. Rudigier, ${ }^{1}$ P. Sarriguren,${ }^{25}$ M. Scheck, ${ }^{6,17}$ M. Seidlitz, ${ }^{1}$ B. Siebeck, ${ }^{1}$ G. Simpson, ${ }^{26}$ P. Thöle, ${ }^{1}$ T. Thomas, ${ }^{1}$ J. Van de Walle, ${ }^{27}$ P. Van Duppen, ${ }^{5}$ M. Vermeulen, ${ }^{8}$ D. Voulot,${ }^{9}$ R. Wadsworth,${ }^{8}$ F. Wenander, ${ }^{9}$ K. Wimmer, ${ }^{4,23}$ K. O. Zell, ${ }^{1}$ and M. Zielinska ${ }^{19}$

${ }^{1}$ Institut für Kernphysik, Universität zu Köln, Zülpicher Strasse 77, D-50937 Köln, Germany

${ }^{2}$ Physics Division, Argonne National Laboratory, Argonne, Illinois, 60439, USA

${ }^{3}$ Department of Physics, University of Tokyo, Hongo, Bunkyo-ku, Tokyo 113-0033, Japan

${ }^{4}$ Physik Department E12, Technische Universität München, D-85748 Garching, Germany

${ }^{5}$ Instituut voor Kern- en Stralingsfysica, Katholieke Universiteit Leuven, B-3001 Leuven, Belgium

${ }^{6}$ Institut für Kernphysik, Technische Universität Darmstadt, D-64289 Darmstadt, Germany

${ }^{7}$ Department of Physics, University of Guelph, Guelph, ON N1G 2W1, Canada

${ }^{8}$ Nuclear Physics Group, Department of Physics, University of York, York YO10 5DD, United Kingdom ${ }^{9}$ CERN, CH-1211 Geneva 23, Switzerland

${ }^{10}$ Department of Physics and Astronomy, University of Rochester, Rochester, New York 14627-0171, USA

${ }^{11}$ Dipartimento di Fisica, Universitá di Camerino, I-62032 Camerino, Italy

${ }^{12}$ CEA, DAM, DIF, F-91297 Arpajon Cedex, France

${ }^{13}$ School of Physics and Astronomy, University of Edinburgh, Edinburgh EH9 3JZ, United Kingdom

${ }^{14}$ Studiecentrum voor Kernenergie/Centre d'Etude de l'énergie Nucléaire (SCK CEN), B-2400 Mol, Belgium

${ }^{15}$ H. Hulubei National Institute of Physics and Nuclear Engineering, Bucharest, Romania

${ }^{16}$ CSNSM-IN2P3-CNSR, Université Paris-Sud 11, F-91405 Orsay, France

${ }^{17}$ Oliver Lodge Laboratory, The University of Liverpool, Liverpool L69 7ZE, United Kingdom

${ }^{18}$ Department of Physics, PO Box 35, FI-40014 University of Jyväskylä, Finland

${ }^{19}$ Heavy Ion Laboratory, University of Warsaw, ul. Pasteura 5A, 02-093 Warszawa, Poland

${ }^{20}$ TRIUMF, 4004 Wesbrook Mall, Vancouver, BC V6T2A3, Canada

${ }^{21}$ Fakultät für Physik, Ludwig-Maximilians-Universität München, D-85748 Garching, Germany

${ }^{22}$ Center for Nuclear Study, University of Tokyo, Hongo, Bunkyo-ku, Tokyo 113-0033, Japan

${ }^{23}$ National Superconducting Cyclotron Laboratory, Michigan State University, East Lansing, Michigan 48824-1321, USA

${ }^{24}$ Departamento de Física Teórica, Universidad Autónoma de Madrid, E-28049 Madrid, Spain

${ }^{25}$ Instituto de Estructura de la Materia, CSIC, Serrano 123, E-28006 Madrid, Spain

${ }^{26}$ Laboratoire de Physique Subatomique et de Cosmologie (LPSC) Grenoble, 53, Rue des Martyrs, F-38026 Grenoble Cedex, France

${ }^{27}$ Kernfysisch Versneller Institut, Universiteit Groningen, NL-9747 AA Groningen, Netherlands (Received 10 November 2011; published 10 February 2012)

The neutron-rich nuclei ${ }^{94,96} \mathrm{Kr}$ were studied via projectile Coulomb excitation at the REX-ISOLDE facility at CERN. Level energies of the first excited $2^{+}$states and their absolute $E 2$ transition strengths to the ground state are determined and discussed in the context of the $E\left(2_{1}^{+}\right)$and $B\left(E 2 ; 2_{1}^{+} \rightarrow 0_{1}^{+}\right)$systematics of the krypton chain. Contrary to previously published results no sudden onset of deformation is observed. This experimental result is supported by a new proton-neutron interacting boson model calculation based on the constrained Hartree-Fock-Bogoliubov approach using the microscopic GognyD1M energy density functional.

DOI: 10.1103/PhysRevLett.108.062701

PACS numbers: 25.70.De, 27.60.+j, 29.30.Kv, 29.38.Gj

Since the availability of high-intensity radioactive ion beams, the extension of the concept of quantum phase transitions to exotic nuclei is of great interest in nuclear physics [1]. Quantum phase transitions occur in atomic nuclei as a function of the number of protons or neutrons and describe changes of the ground-state shapes [2]. The so-called $A \approx 100$ mass region of the nuclear chart around ${ }^{100} \mathrm{Zr}$ is one of the most popular regions for the study of this phenomenon since the zirconium $(Z=40)$ and strontium $(Z=38)$ isotopes undergo a shape transition from almost spherical to strongly deformed shapes when going from neutron number $N=58$ to $N=60$ [3-7]. This 
phenomenon was explained by strongly interacting proton and neutron Nilsson orbitals (cf. [7] and references therein). For the neutrons, the downsloping $\nu 1 / 2^{+}[550]$ and the $\nu 3 / 2^{-}[541]$ orbitals, both resulting from the spherical $\nu h_{11 / 2}$ orbital, drive the deformation. Meanwhile, the extruder $\nu 9 / 2^{+}[404]$ orbital stabilizes the deformation at a saturation level of about $\beta \approx 0.4$ (cf. Ref. [7]), where the parameter $\beta$ represents the axially symmetric deformation [8]. On the other hand, for the protons, the downsloping $\pi 1 / 2^{+}[440]$ and $\pi 3 / 2^{+}[431]$ orbitals, originating from the spherical $\pi g_{9 / 2}$ orbital, are fully occupied at $Z=38$ and $Z=40$, again at a deformation parameter of about $\beta \approx 0.4$. These proton intruder orbitals have a large spatial overlap with the neutron intruder orbitals, creating a minimum in the binding energy at $\beta \approx 0.4$. At the neutron number $N=60$ this deformed configuration is favored over the spherical one and deformation sets in rapidly.

In the krypton chain, only the $\pi 1 / 2^{+}[440]$ proton orbital is fully occupied at $\beta \approx 0.4$. However, the question arises, if the reduced occupation of the deformation driving proton intruder orbitals is still strong enough to enhance deformation as rapidly as in the strontium and zirconium chains. From the experimental point of view, the data around $N=$ 60 in the krypton chain are quite sparse. The $2_{1}^{+}$level energies are well known up to ${ }^{94} \mathrm{Kr}(N=58)$, whereas absolute $E 2$ transition strengths are known only for ${ }^{88} \mathrm{Kr}$ and ${ }^{92} \mathrm{Kr}$ [9]. In 2009, a $\gamma$ ray with an energy of $E_{\gamma}=$ $241 \mathrm{keV}$ was assigned to the $2_{1}^{+} \rightarrow 0_{1}^{+}$transition in ${ }^{96} \mathrm{Kr}$ $(N=60)$ [10]. The sudden drop of the $E\left(2_{1}^{+}\right)$value when going from ${ }^{94} \mathrm{Kr}$ to ${ }^{96} \mathrm{Kr}$ implies a rapid change of the ground-state deformation comparable to those in the strontium and the zirconium chains at $N=60$. On the other hand, the recently published results from mass measurements [11] suggest that the two-neutron separation energies proceed smoothly towards $N=60$, implying a smooth onset of deformation at this neutron number.

To clarify this contradiction, experiments were performed to measure $B\left(E 2 ; 2_{1}^{+} \rightarrow 0_{1}^{+}\right)$values of the exotic even-even nuclei ${ }^{94} \mathrm{Kr}$ and ${ }^{96} \mathrm{Kr}$ employing the technique of sub-barrier projectile Coulomb excitation. The experiments were carried out at the REX-ISOLDE facility at CERN [12,13].

The radioactive nuclei were produced in a fission reaction, induced by a $1.4 \mathrm{GeV}$ proton beam impinging on a high-temperature $\mathrm{UC}_{x}$ primary target. Using the highresolution separator in conjunction with a temperaturecontrolled transfer line a high-purity, low-energy, radioactive $\mathrm{Kr}$-ion beam was produced by the ISOLDE facility and was injected into the REX postaccelerator, accelerating the $\mathrm{Kr}$ ions to about $2.85 \mathrm{MeV} / A$ directed onto a secondary target. Typical particle rates at the secondary target are listed in Table I. The low particle rate in 2011 did not allow the extension of particle- $\gamma$ coincidence analysis, but was crucial for the study of beam composition and enabled
TABLE I. Experimental parameters for the measurements presented in this Letter. For each experimental run the intensity $I_{\mathrm{Kr}}$ and the energy of the $\mathrm{Kr}$ ions at the secondary target $E_{\mathrm{Kr}}$, the measurement duration $t_{\operatorname{Exp}}$ and the ratio of isobaric contamination $R_{\mathrm{Kr} / \mathrm{Rb}}$ are listed.

\begin{tabular}{lccccc}
\hline \hline Isotope & Year & $I_{\mathrm{Kr}}[\mathrm{pps}]$ & $E_{\mathrm{Kr}}[\mathrm{MeV}]$ & $t_{\mathrm{Exp}}[\mathrm{s}]$ & $R_{\mathrm{Kr} / \mathrm{Rb}}$ \\
\hline${ }^{94} \mathrm{Kr}$ & 2009 & $8 \times 10^{4}$ & 267.9 & 60480 & $75(6) / 25(3)$ \\
& 2010 & $4 \times 10^{5}$ & 267.9 & 43560 & $74(7) / 26(4)$ \\
${ }^{96} \mathrm{Kr}$ & 2009 & $4 \times 10^{3}$ & 273.6 & 32760 & $43(4) / 57(6)$ \\
& 2010 & $7 \times 10^{3}$ & 273.6 & 59400 & $46(7) / 54(8)$ \\
& 2011 & $<5 \times 10^{2}$ & 273.6 & & \\
\hline \hline
\end{tabular}

association of observed $\gamma$ rays with nuclei under investigation, as discussed below.

In order to investigate the low-energy level scheme of the projectile particles, the following setup was used. Thin secondary target foils made of $2 \mathrm{mg} / \mathrm{cm}^{2}$ isotopically enriched in either ${ }^{194} \mathrm{Pt}$ to $96.5 \%$ or ${ }^{196} \mathrm{Pt}$ to $97.3 \%$ placed at the center of the spectrometer were bombarded with the radioactive ion beams. The energies of the scattered ejectiles and ${ }^{194,196} \mathrm{Pt}$ recoils were measured with a doublesided silicon strip detector (DSSD) [14]. The subdivision of the DSSD in four quadrants with 16 annular and 12 radial strips per quadrant allowed the measurement of the angular distribution of both the ejectiles and the recoils. In the Coulomb-excitation reaction, both the projectile and the target nuclei may be excited. $\gamma$ rays emitted in the decay back to the ground state were detected with the highefficiency MINIBALL $\gamma$-ray spectrometer [15] consisting of 24 sixfold segmented HPGe detectors. The high granularity of both the DSSD and the MINIBALL spectrometer allowed an event-by-event Doppler correction of the detected $\gamma$ rays. The beam composition was monitored periodically during the experiments with a $\Delta E-E$ telescope, consisting of an ionization chamber filled with $\mathrm{CF}_{4}$ to measure the energy loss, and a silicon detector to measure the residual energy of these particles [16].

Stable beam contaminants like buffer gases from the REX-TRAP and the REX-EBIS were eliminated by applying a software gate to the time $\Delta T_{1}$ between the event and the impact of the proton pulse on the primary target. Additionally, isobaric contaminants caused by the decay during bunching and charge-state breeding processes appeared in the ion beam. Thus, the measured intensity of the target excitation had to be corrected for the ratio between $\mathrm{Kr}$ and the daughter nuclei which was extracted from the $\Delta E-E$ telescope and are listed in Table I.

The Coulomb-excitation cross section of the projectile was determined by normalizing to the cross section of the target, which is well known for ${ }^{194,196} \mathrm{Pt}$. The projectile cross section depends significantly not only on the transitional matrix element $\left\langle 0_{1}^{+}\|M(E 2)\| 2_{1}^{+}\right\rangle$(hereafter referred to as $M_{02}$ ), which is proportional to the $E 2$ transition strength, but also on the diagonal matrix element 
$\left\langle 2_{1}^{+}\|M(E 2)\| 2_{1}^{+}\right\rangle$(hereafter referred to as $M_{22}$ ), which is proportional to the spectroscopic quadrupole moment $Q_{2}$. Since no hint of the excitation of higher-lying states was found in the $\gamma$-ray spectra using the ${ }^{94} \mathrm{Kr}$ and ${ }^{96} \mathrm{Kr}$ beams, higher-lying states were included in the calculations by means of so-called "buffer states" only. The probability of exciting these states via multiple Coulomb excitation is rather unlikely using Pt secondary targets and beam energies of $2.85 \mathrm{MeV} / A$. The annular segmentation of the DSSD allowed the subdivision of the total scattering angle range into sub scattering angle ranges. For the experiments with the ${ }^{94} \mathrm{Kr}$ beams, the size of the subdivisions was chosen to keep the relative statistical uncertainties below values of $2.5 \%$ resulting in three subscattering ranges. Because of the lower statistics in the $\gamma$-ray spectra using the ${ }^{96} \mathrm{Kr}$ beams the same criterion could not be applied, but the full statistics had to be used for calculating the cross sections, resulting in two subscattering ranges corresponding to the scattered projectile, e.g., the scattered target particles.

The matrix elements were determined for each subdivision individually using the coupled-channels computer code GOSIA2 [17]. By giving a fixed set of start parameters for the matrix elements $M_{02}$ and $M_{22}$, theoretical $\gamma$-ray yields are calculated and compared to the experimental ones, qualified by a $\chi^{2}$ value. By varying the initial start parameters for $M_{02}$ and $M_{22}$, the $\chi^{2}$ surfaces with respect to those matrix elements were calculated for each subdivision from 2009 and 2010 individually and summed up in order to obtain the total $\chi^{2}$ surface. By projecting the $1 \sigma$-contour of the total $\chi^{2}$ surface to the respective axes, both matrix elements and their uncertainties were extracted.

Figure 1(a) shows the particle-gated and backgroundsubtracted $\gamma$-ray spectra observed with the ${ }^{94} \mathrm{Kr}$ beam in 2009 (upper panel) and 2010 (lower panel), both Doppler corrected for the projectile mass, i.e., $A=94$. The Doppler-corrected peak at $666.1 \mathrm{keV}$ is associated with
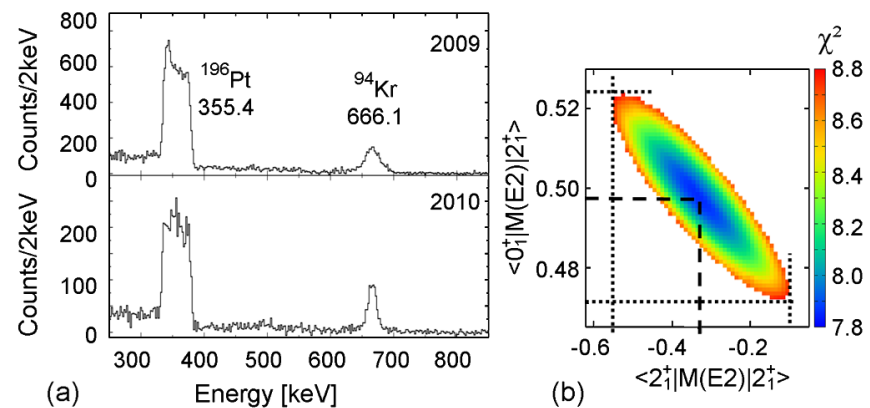

FIG. 1 (color online). (a): Particle-gated and backgroundsubtracted $\gamma$ spectra with the ${ }^{94} \mathrm{Kr}$ beams on the ${ }^{196} \mathrm{Pt}$ target in 2009 (upper panel) and 2010 (lower panel), both Doppler corrected for mass $A=94$. (b): $1 \sigma$ contour of the total $\chi^{2}$ surface with respect to the diagonal and the transitional matrix elements of the $2_{1}^{+}$state in ${ }^{94} \mathrm{Kr}$. the $2_{1}^{+} \rightarrow 0_{1}^{+}$transition in ${ }^{94} \mathrm{Kr}$ [18]. Because of a more precise calibration of the DSSD and repairs of a few MINIBALL cluster segments in 2010, the Doppler correction was improved significantly leading to a reduced FWHM of about $33 \%$ in 2010 . The peak at $355.4 \mathrm{keV}$ corresponding to the $2_{1}^{+} \rightarrow 0_{1}^{+}$transition in the ${ }^{196} \mathrm{Pt}$ target is smeared out due to the Doppler correction using the $\mathrm{Kr}$ mass. From the ratio of the area of these peaks after correction for the relative efficiencies in each spectrum, matrix elements of $M_{02}=0.498(27)$ eb and $M_{22}=$ $-0.34_{-0.22}^{+0.25}$ eb were extracted [cf. Fig. 1(b)]. The $B\left(E 2 ; 2_{1}^{+} \rightarrow 0_{1}^{+}\right)$and $Q_{2}$ values are given in Table II.

Figure 2(a) shows the particle-gated and backgroundsubtracted $\gamma$-ray spectrum with the ${ }^{96} \mathrm{Kr}$ beam in 2009 (upper panel) and 2010 (lower panel), both Doppler corrected for the projectile mass, i.e., $A=96$. Again, the peak at $355.4 \mathrm{keV}$ in the upper panel, which corresponds to the $2_{1}^{+} \rightarrow 0_{1}^{+}$transition in ${ }^{196} \mathrm{Pt}$, and the peak at $329.3 \mathrm{keV}$ in the lower panel, which corresponds to the $2_{1}^{+} \rightarrow 0_{1}^{+}$transition in ${ }^{194} \mathrm{Pt}$, are smeared out due to the Doppler correction using the $\mathrm{Kr}$ mass. The $\gamma$-ray transition that was assigned to the $2_{1}^{+} \rightarrow 0_{1}^{+}$transition at an energy of $E_{\gamma}=$ $241 \mathrm{keV}$ [10] was not observed, but another peak was found at a transition energy of $E_{\gamma}=554.1(5) \mathrm{keV}$. We can exclude the possibilities that the origin of this peak is a stable contamination in the radioactive ion beams due to the applied software gate to the time $\Delta T_{1}$ or a contamination in the secondary target because different secondary targets were used in 2009 and 2010. Thus, this $\gamma$-ray transition corresponds to the decay of an excited state either in ${ }^{96} \mathrm{Kr}$ or ${ }^{96} \mathrm{Rb}$.

To distinguish between these two possibilities, the time dependence of the $554.1 \mathrm{keV} \gamma$-ray intensity was analyzed by fitting an exponential decay function to the time distribution of the $\gamma$-ray intensity using the proton impact on the $\mathrm{UC}_{x}$ primary target as the reference time signal. The decay function is given by the radioactive decay law

$$
N(t)=N_{0} \exp \left[\frac{t}{t_{1 / 2}} \ln (2)\right]
$$

and solely depends either on the half-life of the $\mathrm{Kr}$ nuclei $t_{1 / 2}(\mathrm{Kr})$ or the Rb nuclei $t_{1 / 2}(\mathrm{Rb})$. The function $N(t)$ was fitted to the experimental data by adjusting a constant factor $N_{0}$, which is related to the beam particle flux at the secondary target.

TABLE II. The obtained results for the energies of the $2_{1}^{+}$ states, their E2 transition strengths to the ground state, the lifetimes, and the spectroscopic quadrupole moments.

\begin{tabular}{|c|c|c|c|c|}
\hline Isotope & $\begin{array}{c}E_{\gamma}\left(2_{1}^{+} \rightarrow 0_{1}^{+}\right. \\
{[\mathrm{keV}]}\end{array}$ & $\begin{array}{r}\left(E 2 ; 2_{1}^{+} \rightarrow\right. \\
\quad \text { W.u.] }\end{array}$ & $\tau\left(2_{1}^{+}\right)[\mathrm{ps}]$ & $Q_{2}[\mathrm{~b}]$ \\
\hline $\begin{array}{l}\mathrm{Kr} \\
\mathrm{Kr}\end{array}$ & 666.1(3) & $\begin{array}{l}19.5_{-2}^{+2} \\
334^{+7}\end{array}$ & $12.5_{-1.2}^{+1.5}$ & $-0.45_{-0.30}^{+0.33}$ \\
\hline $\mathrm{Kr}$ & $554.1(5)$ & & $17.9_{-3.3}^{+4.5}$ & $0.26(92)$ \\
\hline
\end{tabular}



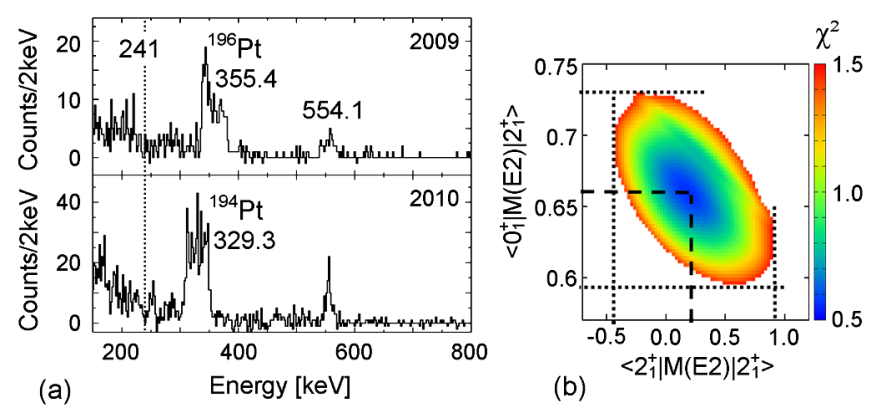

FIG. 2 (color online). (a): Particle-gated and backgroundsubtracted $\gamma$ spectra with the ${ }^{96} \mathrm{Kr}$ beam on the ${ }^{196} \mathrm{Pt}$ target in 2009 (upper panel) and on the ${ }^{194} \mathrm{Pt}$ target in 2010 (lower panel), both Doppler corrected for mass $A=96$. (b): $1 \sigma$ contour of the $\chi^{2}$ surface with respect to the diagonal and the transitional matrix elements of the $2_{1}^{+}$state in ${ }^{96} \mathrm{Kr}$.

In Fig. 3(a), the time structure of the $666.1 \mathrm{keV} \gamma$ ray is shown, corresponding to the $2_{1}^{+} \rightarrow 0_{1}^{+}$transition in ${ }^{94} \mathrm{Kr}$. The solid (green) line indicates the exponential decay function of the ${ }^{94} \mathrm{Kr}$ nuclei, based on a half-life of $t_{1 / 2}\left({ }^{94} \mathrm{Kr}\right)=212(5) \mathrm{ms}$ [18], whereas the dashed (red) line indicates the decay function of the ${ }^{94} \mathrm{Rb}$ nuclei, based on $t_{1 / 2}\left({ }^{94} \mathrm{Rb}\right)=2.702(5) \mathrm{s}$ [18]. The experimental data are well reproduced by the decay function based on the halflife of the ${ }^{94} \mathrm{Kr}$ nuclei.

In Fig. 3(b), the time structure of the $554.1 \mathrm{keV} \gamma$ ray is shown, corresponding either to the $2_{1}^{+} \rightarrow 0_{1}^{+}$transition in ${ }^{96} \mathrm{Kr}$ or a transition in ${ }^{96} \mathrm{Rb}$. The solid (green) line indicates the exponential decay function of the ${ }^{96} \mathrm{Kr}$ nuclei, based on $t_{1 / 2}\left({ }^{96} \mathrm{Kr}\right)=80(7) \mathrm{ms}$ [19], whereas the dashed (red) line indicates the decay function of the ${ }^{96} \mathrm{Rb}$ nuclei, based on $t_{1 / 2}\left({ }^{96} \mathrm{Rb}\right)=203(3) \mathrm{ms}$ [18]. Again, the data are better reproduced by the decay function of the ${ }^{96} \mathrm{Kr}$ nuclei. Thus, the $554.1 \mathrm{keV} \gamma$ peak can be associated with the $2_{1}^{+} \rightarrow 0_{1}^{+}$transition in ${ }^{96} \mathrm{Kr}$.

With the new assignment of the $554.1 \mathrm{keV} \gamma$ ray to the $2_{1}^{+} \rightarrow 0_{1}^{+}$transition in ${ }^{96} \mathrm{Kr}$, values for the matrix elements of $M_{02}=0.66(7)$ eb and $M_{22}=0.2(7)$ eb were extracted [cf. Fig. 2(b)]. The corresponding $B\left(E 2 ; 2_{1}^{+} \rightarrow 0_{1}^{+}\right)$and $Q_{2}$ values are given in Table II.

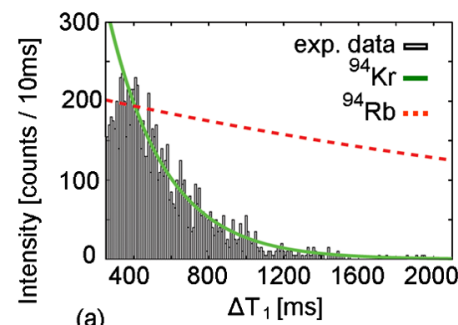

(a)

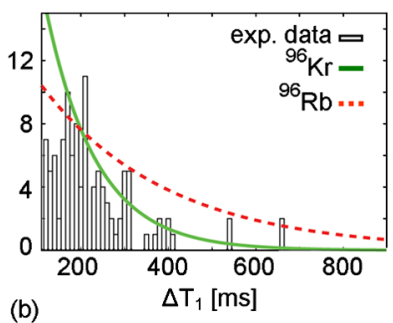

FIG. 3 (color online). Time structures of the $666.1 \mathrm{keV} \gamma$ ray corresponding to the $2_{1}^{+} \rightarrow 0_{1}^{+}$transition in ${ }^{94} \mathrm{Kr}$ (a) and the $554.1 \mathrm{keV} \gamma$ ray corresponding either to the $2_{1}^{+} \rightarrow 0_{1}^{+}$transition in ${ }^{96} \mathrm{Kr}$ or an unknown transition in ${ }^{96} \mathrm{Rb}(\mathrm{b})$.
In Fig. 4, the new $E\left(2_{1}^{+}\right)$systematics and the extended $B\left(E 2 ; 2_{1}^{+} \rightarrow 0_{1}^{+}\right)$systematics of the krypton $(Z=36)$ chain are plotted. With the $E\left(2_{1}^{+}\right)$value for ${ }^{96} \mathrm{Kr}$ and the new $B\left(E 2 ; 2_{1}^{+} \rightarrow 0_{1}^{+}\right)$in ${ }^{94,96} \mathrm{Kr}$, determined in this Letter, both systematics imply a smooth onset of deformation at $N=60$, which is consistent with the conclusions from recent mass measurements [11] and results obtained in isotopic shift $\delta\left\langle r_{c}^{2}\right\rangle$ measurements [20]. The latter was discussed theoretically in Ref. [21], where the smooth behavior in $\mathrm{Kr}$ isotopes was interpreted as a stabilization of the oblate shapes along the isotopic chain. This interpretation is supported by the negative sign of the spectroscopic quadrupole moment in ${ }^{94} \mathrm{Kr}$ indicating an oblate ground-state shape. The large uncertainty of the spectroscopic quadrupole moment in ${ }^{96} \mathrm{Kr}$ also allows a negative sign, but for a qualitative discussion, this value has to be determined more precisely.

The assignment of the $554.1 \mathrm{keV} \gamma$ ray is supported by new calculations within the proton-neutron interacting boson model (IBM-2) [22-26]. The IBM-2 employs the proton (neutron) monopole $s_{\pi}\left(s_{\nu}\right)$ and the quadrupole $d_{\pi}\left(d_{\nu}\right)$ bosons, which reflect the collective $J=0^{+}$and $2^{+}$pairs of valence protons (neutrons), respectively $[25,26]$. We exploit the IBM-2 because it is more associated with a microscopic picture than the original version of IBM without distinction between protons and neutrons. The parameters of the IBM-2 Hamiltonian based on a ${ }^{78} \mathrm{Ni}$ core are determined following the procedure of Ref. [27]: the constrained self-consistent mean-field energy surface based on a given energy density functional (EDF) is mapped onto the expectation value of the boson Hamiltonian in the coherent-state formalism [28].

The calculations provided level energies of the excited $\mathrm{O}_{2}^{+}, 2_{1}^{+}, 2_{2}^{+}$, and $4_{1}^{+}$states and $E 2$ transition strengths in the neutron-rich isotopes ${ }^{86-96} \mathrm{Kr}$. The results are shown in Fig. 5, compared to the experimental data. The excitation energies were calculated, without any adjustment to the experimental results. The IBM-2 Hamiltonian of the form used, e.g., in Ref. [29] is derived from the (constrained) Hartree-Fock-Bogoliubov method using the Gogny-D1M EDF [30]. The calculated $2_{1}^{+}$level energies reproduce the

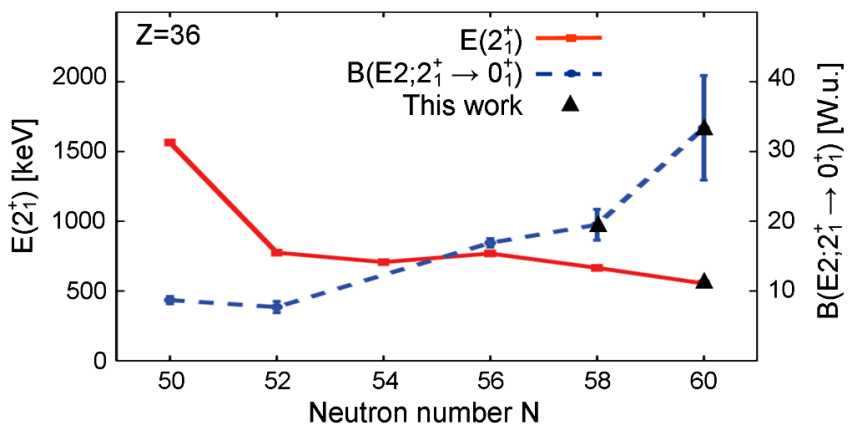

FIG. 4 (color online). $\quad E\left(2_{1}^{+}\right)$(solid red line) and $B\left(E 2 ; 2_{1}^{+} \rightarrow\right.$ $\mathrm{O}_{1}^{+}$) (dotted blue line) systematics of the $\mathrm{Kr}$ isotopes. 


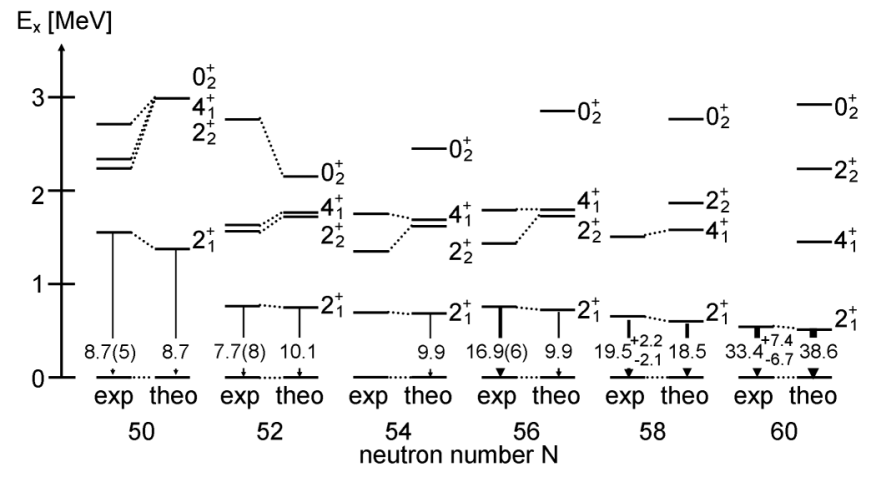

FIG. 5. Experimental and theoretical excitation energies of excited $0_{2}^{+}, 2_{1}^{+}, 2_{2}^{+}$, and $4_{1}^{+}$states in the $\mathrm{Kr}$ isotopes with $50 \leq$ $N \leq 60$. The experimental $B\left(E 2 ; 2_{1}^{+} \rightarrow 0_{1}^{+}\right)$values are taken from $[9,31]$ and this Letter and are given in W.u.

experimental data up to $N=60$ very well. At $N=60$, the calculated $2_{1}^{+}$level energy of $534 \mathrm{keV}$ supports our assignment of the $554.1 \mathrm{keV} \gamma$ ray to the $2_{1}^{+} \rightarrow 0_{1}^{+}$transition in ${ }^{96} \mathrm{Kr}$. Furthermore the calculated level energies of the higher-lying states also reproduce the experimental data well.

For the calculation of the $E 2$ transition strengths, the standard $E 2$ transition operator was used, given by $T^{(E 2)}=$ $e_{\pi} \hat{Q}_{\pi}+e_{\nu} \hat{Q}_{\nu}$ [24]. The effective proton-boson charge $e_{\pi}=0.07 \mathrm{eb}$ was adjusted to reproduce the experimental $B\left(E 2 ; 2_{1}^{+} \rightarrow 0_{1}^{+}\right)$value in ${ }^{86} \mathrm{Kr}(N=50)$, taken from Ref. [31]. The effective neutron-boson charge was set to $e_{\nu}=0 \mathrm{eb}$ in order to reduce the number of free parameters (cf. [32,33]). The experimental $B(E 2)$ values are nicely reproduced by the theoretical ones, also implying a smooth increase of collectivity (see Fig. 5) in the neutron-rich $\mathrm{Kr}$ isotopes towards $N=60$.

To summarize, new experimental results on the first excited $2^{+}$states in the neutron-rich $\mathrm{Kr}$ isotopes ${ }^{94} \mathrm{Kr}$ and ${ }^{96} \mathrm{Kr}$ are presented, which resolve the conflict between the results from Refs. $[10,11,20]$. The investigated nuclei were excited via projectile Coulomb excitation. Contrary to what was found in Ref. [10], the $241 \mathrm{keV} \gamma$ ray, interpreted as the $2_{1}^{+} \rightarrow 0_{1}^{+}$transition in ${ }^{96} \mathrm{Kr}$, was not observed. By analyzing the time structure of the $554.1 \mathrm{keV} \gamma$ ray measured in the present experiment, we assigned this $\gamma$ ray to the $2_{1}^{+} \rightarrow 0_{1}^{+}$transition in ${ }^{96} \mathrm{Kr}$. Absolute E2 transition strengths of the $2_{1}^{+} \rightarrow 0_{1}^{+}$transitions in ${ }^{94} \mathrm{Kr}$ and ${ }^{96} \mathrm{Kr}$ are determined using the computer code GOSIA2 [17]. The $\gamma$ spectroscopic results imply a smooth onset of deformation in the neutron-rich krypton isotopes around $N=60$, which is in agreement with the results obtained in mass measurements [11]. This interpretation is strongly supported by our new calculations with the IBM-2 Hamiltonian determined based on the microscopic Gogny-D1M EDF.

This work was supported by the BMBF under Contracts No. 06KY9136 and No. 06MT238, by ENSAR, by the DFG Cluster of Excellence Origin and Structure of the
Universe, and by the grant-in-aid for scientific research for JSPS Fellows (Grant No. 217368). One of the authors (J.D.) would like to acknowledge the support of FWOVlaanderen (Belgium).

*malbers@ikp.uni-koeln.de

[1] R. F. Casten and E. A. McCutchan, J. Phys. G 34, R285 (2007).

[2] P. Cejnar, J. Jolie, and R. F. Casten, Rev. Mod. Phys. 82 2155(2010).

[3] P. Federmann and S. Pittel, Phys. Lett. B 69, 385 (1977).

[4] Nuclear Structure of the Zr Region, edited by J. Eberth, R. A. Meyer, and K. Sistemich (Springer, Berlin, 1988).

[5] G. Lhersonneau et al., Z. Phys. A 330, 347 (1988).

[6] M. Hotchkis et al., Phys. Rev. Lett. 64, 3123 (1990).

[7] W. Urban et al., Eur. Phys. J. A 22, 241 (2004).

[8] A. Bohr and B. R. Mottelson Nuclear Structure(Benjamin, New York, 1975) Vols. I and II.

[9] D. Mücher, Ph.D. thesis, Universität zu Köln, 2009, http:// kups.ub.uni-koeln.de/2868/.

[10] N. Marginean et al., Phys. Rev. C 80, 021301(R) (2009).

[11] S. Naimi et al., Phys. Rev. Lett. 105, 032502 (2010).

[12] D. Habs et al., Nucl. Instrum. Methods Phys. Res., Sect. B 139, 128 (1998).

[13] O. Kester et al., Nucl. Instrum. Methods Phys. Res., Sect. B 204, 20 (2003).

[14] A. Ostrowski et al., Nucl. Instrum. Methods Phys. Res., Sect. A 480, 448 (2002).

[15] J. Eberth et al., Prog. Part. Nucl. Phys. 46, 389 (2001).

[16] J. Van de Walle et al., Phys. Rev. C 79, 014309 (2009).

[17] T. Czosnyka, D. Cline, and C. Wu, Bull. Am. Phys. Soc. 28, 745 (1983).

[18] http://www.nndc.bnl.gov, (2011).

[19] U. Bergmann et al., Nucl. Phys. A714, 21 (2003).

[20] M. Keim et al., Nucl. Phys. A586, 219 (1995).

[21] R. Rodriguez-Guzman, P. Sarriguren, and L. M. Robledo, Phys. Rev. C 83, 044307 (2011).

[22] A. Arima and F. Iachello, Phys. Rev. Lett. 35, 1069 (1975).

[23] A. Arima et al., Phys. Lett. B 66, 205 (1977).

[24] A. Arima and F. Iachello, The Interacting Boson Model (Cambridge University Press, Cambridge, England, 1987).

[25] T. Otsuka et al., Phys. Lett. B 76, 139 (1978).

[26] T. Otsuka, A. Arima, and F. Iachello, Nucl. Phys. A309, 1 (1978).

[27] K. Nomura, N. Shimizu, and T. Otsuka, Phys. Rev. Lett. 101, 142501 (2008).

[28] A.E. L. Dieperink, O. Scholten, and F. Iachello, Phys. Rev. Lett. 44, 1747 (1980).

[29] K. Nomura, T. Otsuka, R. Rodriguez-Guzman, L. M. Robledo, and P. Sarriguren, Phys. Rev. C 83, 014309 (2011).

[30] S. Goriely, S. Hilaire, M. Girod, and S. Peru, Phys. Rev. Lett. 102, 242501 (2009).

[31] T. J. Mertzimekis et al., Phys. Rev. C 64, 024314 (2001).

[32] N. Pietralla et al., Phys. Rev. C 58, 796 (1998).

[33] C. Fransen et al., Phys. Rev. C 67, 024307 (2003). 